\title{
Role of Interleukin-6 Correlated to C. pneumoniae Infection as a Biomarker for Prediction of Stroke Severity in Young Patients with Acute Ischemic Stroke
}

\author{
M. V. Padma Srivastava, ${ }^{1}$ Ashu Bhasin, ${ }^{1}$ Rama Chaudhry, ${ }^{2}$ Sakshi Sharma, ${ }^{1}$ \\ S. Vivekanandhan, ${ }^{3}$ Rohit Bhatia, ${ }^{1}$ and Manjari Tripathi ${ }^{1}$ \\ ${ }^{1}$ Department of Neurology, AIIMS, New Delhi, India \\ ${ }^{2}$ Department of Microbiology, AIIMS, New Delhi, India \\ ${ }^{3}$ Department of Neuro-Biochemistry, AIIMS, New Delhi, India
}

Correspondence should be addressed to M. V. Padma Srivastava; vasanthapadma123@gmail.com

Received 18 January 2014; Revised 3 June 2014; Accepted 29 June 2014; Published 20 July 2014

Academic Editor: Taku Sugawara

\begin{abstract}
Copyright ( 2014 M. V. Padma Srivastava et al. This is an open access article distributed under the Creative Commons Attribution License, which permits unrestricted use, distribution, and reproduction in any medium, provided the original work is properly cited.
\end{abstract}

Background. Stroke in young is the leading cause of morbidity and mortality in the Indian subcontinent with a reported incidence of $15-30 \%$ of all stroke patients. The mechanisms for stroke in the young may include unconventional risk factors such as infections. Causative role of C. pneumoniae infection in patients with acute ischemic stroke (AIS) remains unresolved till date, although the link between C. pneumoniae and cerebrovascular disease has been investigated in many studies. This study examined the upregulation of IL-6 after acute cerebral ischemia and correlated the same with the C. pneumoniae antibody titres (IgG, IgA, and IgM). Methods. We studied blood samples from eighty $(n=80)$ acute stroke patients and healthy age- and sex-matched controls. Venous blood samples were drawn within one week from the onset of stroke. Detection of IgA, IgG, and IgM antibodies to C. pneumoniae was done with a validated microimmunofluorescence (MIF) technique from $5 \mathrm{~mL}$ of serum in all subjects. Interleukin-6 was estimated with sandwich ELISA method. Results. The IL-6 levels were elevated in patients with a mean $28.9 \pm 8.6 \mathrm{pg} / \mathrm{mL}$ as compared to $4.7+$ $1.8 \mathrm{pg} / \mathrm{mL}$ in healthy age-matched controls (95\% CI: 37.7 to 78.4; $P=0.004)$. On correlation of IL-6 to stroke severity, it was found that 30 patients with NIHSS between 0 and 15 had mean IL- 6 of $24.6 \mathrm{pg} / \mathrm{mL}$ and 50 patients had NIHSS of 18.8 (severely affected) with a mean IL-6 of $43.8 \mathrm{pg} / \mathrm{mL}$. On multivariate analysis after adjusting for sex, hypertension, diabetes mellitus, smoking, and alcohol, the IgA seropositivity yielded an adjusted OR for stroke (4.72; 95\% CI: 1.61, 13.83; $P=0.005$ ), while IgG seropositivity did not show a statistically significant result. We also observed that $81 \%$ of cases were seropositive for IgA versus $32 \%$ of controls $(P=0.003)$ followed by IgG, as $52.7 \%$ of cases were seropositive versus $17.3 \%$ of controls $(P=0.05)$. Multiple regression analysis was done with IL-6 as dependent variable to antibody with IL-6 as dependent variable to Cp-IgA, Cp-IgG, and IgM with $10.4 \%$ change in the IL-6 titres showing statistical significant result $F(4,115)=3.32, P=0.01$. Conclusions. IL- 6 has important role after acute ischemic stroke and is correlated with stroke severity and may correlate to acute or chronic infectious states with C. pneumonia.

\section{Introduction}

Young stroke patients constitute $15-30 \%$ of all stroke patients in India, to that of $3.0-8.5 \%$ of all stroke patients reported from the West [1]. Stroke in young, including stroke in children and young adults ( $<45$ years), is an important cause of morbidity throughout the world, especially in developing countries [2]. The initial data from the Indian Collaborative
Acute Stroke Study (ICASS) performed under the aegis of World Health Organization (WHO), supports a high prevalence of vascular risk factors and a relatively younger age of stroke cases in India [3-5]. Recognition of trends of stroke burden in India is essential to assist target prevention and management strategies.

Atherosclerosis is a leading cause of mortality and morbidity worldwide whereas the known conventional risk 
factors including hypertension, cigarette smoking, diabetes mellitus, and hyperlipidemia do not fully explain the incidence of ischemic stroke in the young age group of 1845 years [6, 7]. Inflammation has been postulated to play an important role in the initiation and development of atherosclerosis [8]. Recently, increasing interest has focused on the putative causal role of chronic infections such as Helicobacter pylori [9] and C. pneumoniae (Cp) in acute stroke pathophysiology. C. pneumoniae is a Gram-negative intracellular bacterium that commonly causes upper respiratory tract infections [10]. Proinflammatory cytokines, oxidized low-density lipoproteins, interleukin-1 (IL-1, 6) [11], tumor necrosis factor- $\alpha$ (TNF- $\alpha$ ), selectins, inflammatory stimuli like interleukin-6 (IL-6), C-reactive protein (CRP), and acute-phase reactants [12] are potential mediators which help to identify and monitor the ongoing inflammatory response to injury/ischemia.

Several retrospective and cross-sectional studies have shown association between previous infections with $C$. pneumoniae, herpes simplex virus (HSV), Cytomegalovirus (CMV), hepatitis A (HAV) [13], and other respiratory and oral pathogens with cardiovascular and cerebrovascular diseases. A panel of inflammatory markers has shown association with coronary artery disease in some studies reported from the West [14]. Causative role of C. pneumoniae infection in patients with acute ischemic stroke (AIS) remains unresolved till date, although the link between C. pneumoniae and cerebrovascular disease has been investigated in a number of seroepidemiological studies [15]. The source of increased cytokines in stroke is another matter of controversy, as they are known to be secreted by cells of the central nervous system and leukocytes. IL-6 is an inflammatory biomarker and its increased production is associated with disturbances of homeostasis such as inflammatory diseases of vessel wall, trauma, or sepsis [16]. The objective of the present study was to assess the prevalence of inflammatory biomarker interleukin-6 (IL-6) in young patients with acute stroke and to correlate them with IgG, IgM, and IgA antibody titers of $C$. pneumoniae.

\section{Methods}

This study was prospective observational case control design performed at a tertiary care hospital with specialized acute stroke care. Young patients (18-45 years), diagnosed with acute ischemic stroke (AIS) within one week of index event, were recruited into the study. Complete medical history of hypertension, smoking, diabetes mellitus, hypercholesterolemia, ischemic heart disease, stroke, or TIA was recorded with neurological examination included recording of National Institute of Health for Stroke Severity (NIHSS). CT head was performed in all and if needed MRI/CTA/MRA was performed according to the clinical requirement. Controls selected for the above analysis were healthy age- and sex-matched subjects, who did not have any history of cerebrovascular, neurological, or long standing medical illness. Blood samples were drawn within one week from the onset of stroke. In each case, $5 \mathrm{~mL}$ of serum was obtained by centrifugation at $+4^{\circ} \mathrm{C}$ and stored at $-70^{\circ} \mathrm{C}$ until analysis. Detection of IgA, IgG, and IgM antibodies to C. pneumoniae was done with a validated microimmunofluorescence (MIF) technique. The cutoff point for seropositivity was $1 / 16$ for IgA and 1/64 for IgG. Inflammatory biomarker IL-6 was determined using Immulite Kit. Detection of Cp-IgA, Cp$\mathrm{IgG}$, and $\mathrm{Cp}$-IgM antibodies was done with validated quantitative sandwich enzyme immunoassays according to the manufacturer's instructions ( $\mathrm{R} \& \mathrm{D}$ Systems, Minneapolis, MN, USA).

\section{Statistical Methods}

All serological results were dichotomized as positive or negative. Simultaneously, an alpha level of 0.05 was set as the level of significance. SPSS version 12.0 for Windows (SPSS Inc., Chicago, IL, USA) was used for statistical analysis. For continuous variables such as age, NIHSS, and calculation of IL-6, descriptive statistics was calculated and reported as means \pm SD. Normality of distribution of continuous variables was verified using the parametric tests.

\section{Results}

Eighty $(n=80)$ AIS patients with the mentioned inclusion criteria with eighty healthy $(n=80)$ age- and sex-matched controls were recruited for the study. The mean age in study group was $43.6 \pm 6.7$ years (range 25 to 45 ) and $43.2 \pm 7.3$ years in controls with diabetes mellitus $23.4 \%$, hypercholesterolemia $35.4 \%$, and smoking $22.3 \%$. There were no cardiovascular risk factors in the control group. The sensitivity of the minimum detectable dose of IL-6 was $0.09 \mathrm{pg} / \mathrm{mL}$. In all our patients, values for IL- 6 were above the highest standard of $7 \mathrm{pg} / \mathrm{mL}$ and were then recorded as $>7 \mathrm{pg} / \mathrm{mL}$ as positive and less as negative. The IL-6 levels were elevated in patients with a mean $28.9+8.6 \mathrm{pg} / \mathrm{mL}$ as compared to $4.7+1.8 \mathrm{pg} / \mathrm{mL}$ in healthy age-matched controls $(95 \% \mathrm{CI}$ : 37.7 to $78.4 ; P=0.004)$. On correlation of IL-6 to stroke severity, it was found that 30 patients with NIHSS between 0 and 15 had mean IL- 6 of 24.6 and 50 patients had NIHSS of 18.8 (severely affected) with a mean IL- 6 of 43.8 (Figure 1).

From our previous cohort [17], out of 51 patients to that of 48 controls, $\mathrm{Cp}$-IgA seropositivity was significantly increased and associated with acute ischemic stroke (unadjusted odds ratio: 3.1 ; 95\% CI: $1.38,6.96 ; P=0.005)$, whereas $\mathrm{Cp}-\mathrm{IgG}$ (unadjusted OR 0.44; 95\% CI: 0.18, 1.09; $P=0.07$ ) and CpIgM (unadjusted OR: 1.1 ; 95\% CI: $0.36,3.3 ; P=0.88$ ) did not. There was no difference in IgA or IgG positivity in different stroke subtypes. On multivariate analysis after adjusting for sex, hypertension, diabetes mellitus, smoking, and alcohol, the IgA seropositivity yielded an adjusted OR for stroke (4.72; 95\% CI: $1.61,13.83 ; P=0.005)$, while IgG seropositivity did not show a statistically significant result.

In our patients, $81 \%$ of cases were seropositive for IgA versus $32 \%$ of controls $(P=0.003)$ (Figure 2$)$. This difference was less striking for IgG, as $52.7 \%$ of cases were seropositive versus $17.3 \%$ of controls $(P=0.05)$. When considering both $\operatorname{IgA}$ and $\operatorname{IgG}$ indices, the percentage of stroke patients 


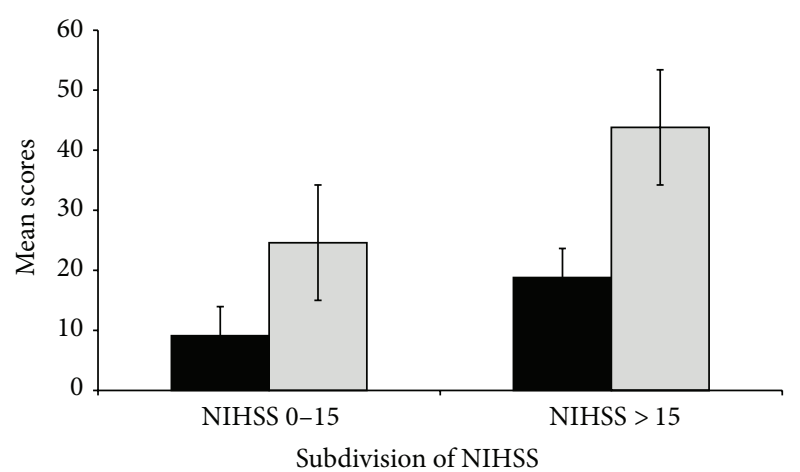

- NIHSS

$\square$ IL-6

Figure 1: Mean IL-6 and mean NIHSS scores in moderate and severely affected patients.

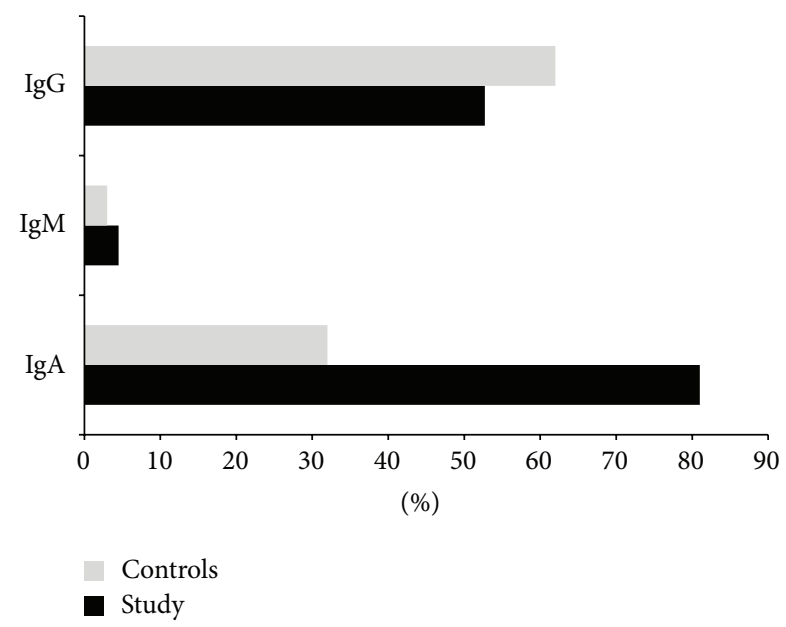

Figure 2: Percentage of seropositivity of Cp-IgA, IgM, and IgE in study (patients) versus controls.

with serologic evidence of $C$. pneumoniae infection was significantly higher $(71 \%)$ than in controls $(20 \% ; P<0.01)$. IgM antibodies were found to be similar and negative in patients as well in controls. This variation was not found to be statistically significant between patients and controls $(P>0.05)$. Multiple regression analysis was done with IL6 as dependent variable to antibody with IL- 6 as dependent variable to $\mathrm{Cp}-\operatorname{IgA}$, Cp-IgG, and $\operatorname{IgM}$ with $10.4 \%$ change in the IL-6 titres to the other variables showing statistical significant result $F(4,115)=3.32, P=0.01$ (Figure 3 ).

\section{Discussion}

The aim of this study was to study the upregulation of IL-6 within a week post-AIS and determine the possible relationship between IL-6 and stroke severity and to Cp-IgA, IgE, and IgG antibody titers.

Interleukin-6 (IL-6) is an inflammatory biomarker, whose increased production is associated with disturbances of homeostasis, such as trauma, sepsis, or inflammatory

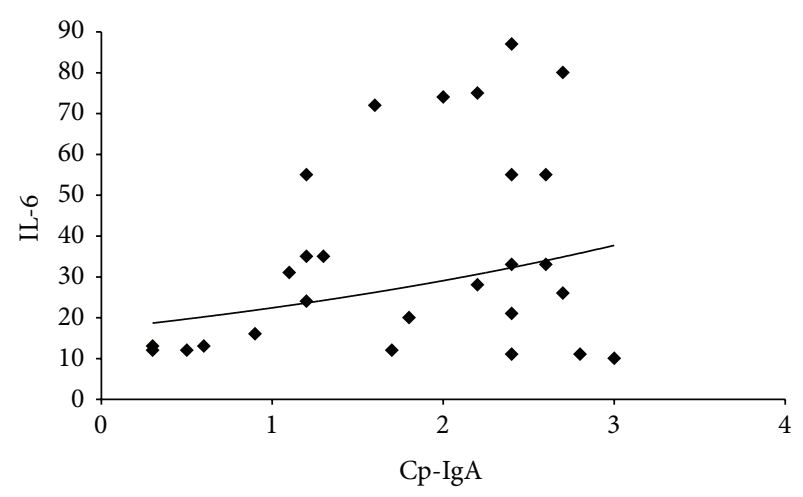

Figure 3: Correlation of IL-6 to Cp IgA antibodies. A strong correlation was observed $(r=0.78 ; P=0.01)$ in patients with high IgA titres $>1$ have high IL- 6 levels.

diseases. Waje-Andreassen et al. reported that IL-6 levels were increased in the acute phase of stroke compared with healthy controls $(P=0.002)$ and correlated with larger stroke volume $(P=0.012)$ and less favorable prognosis after 1 year, measured by ESS $(P=0.014)$ and BI $(P=0.006)$. This study was conducted on eleven stroke patients with symptoms over one year [11]. We correlated IL-6 levels with the severity of stroke and found that greater levels of IL-6 (mean $45.2 \mathrm{pg} / \mathrm{mL}$ ) was highly correlated with greater NIHSS scores $(r=0.89 ; P=0.002)$ (Figure 1) suggesting putative role of IL-6 in the cellular and molecular inflammatory cascade of cerebral ischemia.

Clinical and experimental data has shown that leukocyte recruitment is a hallmark that occurs over hours to days after cerebral ischemia. In mouse models of transient MCAO, elevated IL-1 $\beta$ mRNA expression occurs within the first $15-30$ min after permanent MCAO and elevated IL-1 $\beta$ protein expression occurs a few hours later and remains elevated for up to 4 days $[12,14]$. Flow cytometric examination of the inflammatory cell infiltration in the ischemic brain reveals that (CD3) $\mathrm{T}$ cells increased relatively late, 3-4 days after ischemia, whereas activated CD11b, microglia/macrophages, and neutrophils increased significantly early. The expression of IL-23 (most likely derived from activated microglia/macrophages) increases on day 1 after reperfusion injury, whereas IL-17 levels are elevated after day 3 , and its induction was dependent on IL-23 [18].

IL- 6 is a key regulator of the acute-phase response and elevates the temperature set point in the hypothalamus and stimulates the expression of other acute-phase proteins in the liver $[19,20]$. It has been well demonstrated that IL6 is produced locally by resident brain cells for poststroke angiogenesis in experimental models of stroke [21]. At 2nd day after mild transient brain ischemia, there was an increase in IL-6 gene transcription and protein levels in ischemic brain that was accompanied by a significant early upregulation of angiogenesis-associated gene [22]. Genetic polymorphism of IL- 6 has been studied and its expression is mainly regulated at the transcriptional level [23]. The promoter of the human IL-6 gene contains several polymorphisms that influence IL6 gene transcription in selected cell types inside the brain. In 
a randomized trial for acute stroke, IL-1R (receptor), readily crossed the blood-brain barrier, was safe to use and seemed to afford some benefit, particularly for patients with cortical infarcts [24].

Acalovschi et al. [25] found that IL-6 levels in patients with ischemic stroke were dependent not only on the infarct size, but also on the haplotype of IL-6 promoter region. However, stroke is a complex multifactorial disease in which several genes and environmental factors are important role models [26]. The infarct size was not calculated from our results and hence could not be correlated with the biomarker levels.

The source of the increased IL- 6 levels detected in the systemic circulation in the acute phase of ischemic stroke is unknown. As IL-6 is correlated with the stroke volume, it is possible that endothelial cells, microglia, astrocytes, and leukocytes are responsible for the cytokine production [27]. IL- 6 and IL- 6 mRNA have been found in the pyramidal cells of the hippocampus, and it is therefore possible that damaged neurons also contribute to the increased IL-6 levels. An intrathecal synthesis of IL- 6 is supported by previous results showing peak levels of IL-6 in the CSF 2-3 days after stroke onset [28].

IL-6 effects on leukocyte adhesion, infiltration, and activation have been vastly studied [28]. Hahn et al. demonstrated by flow cytometric analysis the infiltration of $\mathrm{T}$ lymphocytes in mouse brain $72 \mathrm{~h}$ after transient middle cerebral artery occlusion [29]. However, we did not find any correlation with total number of leucocytes (TLC) and differential leucocyte counts (DLC) in peripheral blood smear, although reports suggest that there is a strong association of IL- 6 with an increased TLC and DLC following cerebral ischemia [30].

Cp-IgG antibodies may be considered as markers of "chronic" infection and they could be in circulation for 3-5 years following infection. The IgA antibodies may be considered as markers of a primary, acute, or chronic infection and stay in circulation on an average for 3-5 days or longer $[31,32]$. Several studies have demonstrated the presence of $C$. pneumoniae in atherosclerotic lesions in coronary arteries, aorta, and carotid arteries obtained from autopsy and end-arterectomy specimens [33]. One Indian study which has shown association of high C-reactive protein (CRP) in stroke patients lends further credence to the hypothesis that infection with $C$. pneumoniae, acute or chronic, may be associated with atherosclerosis through the mechanism of low-grade inflammation [34].

The important group of factors significantly elevating IL6 level constitutes vascular risk factors: hypercholesterolemia and hypertension followed by obesity and cigarette smoking. In our report, patients who had previous history of cardiac disease $(29.1 \%)$ and higher cholesterol levels (29.4\%) had greater IL-6 levels as compared to the other risk factors which may indicate that an increased levels of IL-6 is a silent marker of coronary events and thereby a risk factor for stroke (OR, 95\% CI: 37.7 to $78.4 ; r=0.87 ; P=0.002$ ).

One of the major limitations of the study was that acute stroke subtype was not done and hence could not be correlated according to ASCO or TOAST score. In relation to age and gender, no difference was detected between the groups. Such data corroborate the previous report which did not find any difference between young people and the elderly or men and women [35].

Multiple regression analysis was done with IL-6 as dependent variable to antibodies $\operatorname{IgA}, \operatorname{IgG}$, and $\operatorname{IgM}$ with $32.4 \%$ sensitivity in IL-6 titres to Cp-IgA $(F(4,115)=3.32, P=$ $0.01)$. In our patients IL-6 was recorded at 1st day (35 patients), 2nd day (20 patients), and 4 th day (25 patients) and it was by chance that we found out that mean IL- 6 was high in patients whose measurement were taken at 4th day as compared to those taken at 1st and 2nd day which is in congruence with the reports stating that IL-6 peaks after 3 rd day in cerebral ischemia [36-38].

The infection caused by $C$. pneumoniae has been described as an independent risk factor for cerebrovascular diseases; however, it is still under debate whether the microorganism would be the causal agent to the ones that believe it would merely be an innocent bystander. Despite the strong association found through several epidemiologic and experimental studies, the causality of the facts cannot be simply established. The Northern Manhattan study [35], suggested that chronic C. pneumoniae infection may be a risk factor for stroke in the elderly as well as the young. Also Cpantibody levels could rise after stroke primarily because of immune response to common epitopes in the infarcted brain tissue (i.e., "molecular mimicry") or nonspecific immunological activation $[39,40]$. Future prospects of the same study would be to evaluate whether C. pneumonia is causative organism, to study the titres at one week, one month, and 3 months (one time sample collection), or to investigate the titres repeatedly in one patient after a week, two weeks, and one month.

The pathogenesis of cerebral brain ischemia in the young has been implicated as a result of dissection of extracranial vessels, hyperhomocysteinemia, cardiac causes, and immunological disturbances. Accumulations of erythrocytes is known to be the initial step in the pathological cascade, which is followed by blood-brain barrier (BBB) disarray finally leading to microbleeds that cause necrotic tissue damage in terms of microinfarcts. Dissection of the carotid or vertebral arteries is associated with disorders of connective tissue such as Ehlers-Danlos syndrome, Marfan's syndrome, pseudoxanthomas, and fibromuscular dysplasias. There is an association with intracranial aneurysms and possibly with alpha-l-antitrypsin deficiency. Lupus anticoagulant and anticardiolipin antibodies have activity against protein-phospholipid complexes. They are detected in 50\% of patients with lupus and other autoimmune disorders such as rheumatoid arthritis and giant cell arteritis leading to another important cause of ischemia in younger population. The most common cardiac lesions are rheumatic valve disease, bacterial endocarditis, cardiomyopathies, ischemic dyskinetic segments, patent foramen ovale, and so forth [41].

It is hypothesized that stroke outcomes may be improved by antileukocyte strategies (including antiadhesion molecule strategies), which are targeted specifically to the reperfusion period [42, 43]. Despite intense investigations, it remains unclear why anti-inflammatory therapy succeeded in animal 
models but not in clinical applications in management of acute stroke. Experimental studies demonstrated that antileukocyte strategies may extend the therapeutic time window of tPA reperfusion therapy in acute stroke [44].

\section{Summary and Conclusion}

Raised IgA antibodies as compared to IgM and IgG indicating probably recurrent acute infections may have set the stage for the acute ischemic events with other atherosclerotic risk factors in these patients [43-45]. These results are supported by the fact that inflammation and immune reaction secondary to acute infection with $C$. pneumoniae may trigger an acute stroke. We acknowledge the limitations of our study: infarct volume, CRP levels were not measured, stroke subtype and serial measurements of IL-6 were not recorded in a single patient. Third generation tetracyclines, for example, minocycline is an attractive attempt to promote neuroprotective effects after stroke, especially in a developing country where infection and cerebrovascular disease contribute significantly to health mortality and morbidity. It would be conclusive to state that IL-6 has important role after acute ischemic stroke and is correlated with stroke severity and may correlate to acute or chronic infection with C. pneumonia.

\section{Conflict of Interests}

The authors declare that there is no conflict of interests regarding the publication of this paper.

\section{Acknowledgment}

The study is funded by the Indian Council of Medical Research (ICMR), New Delhi, India.

\section{References}

[1] P. M. Dalal, "Strokes in young and elderly: risk factors and strategies for stroke prevention," Journal of Association of Physicians of India, vol. 45, no. 2, pp. 125-131, 1997.

[2] T. K. Banerjee and S. K. Das, "Epidemiology of stroke in India," Neurology Asia, vol. 11, pp. 1-4, 2006.

[3] M. S. V. Elkind, M. L. C. Tondella, D. R. Feikin, B. S. Fields, S. Homma, and M. R. Di Tullio, "Seropositivity to Chlamydia pneumoniae is associated with risk of first ischemic stroke," Stroke, vol. 37, no. 3, pp. 790-795, 2006.

[4] J. Abraham, P. S. Rao, S. G. Inbaraj, G. Shetty, and C. J. Jose, "An epidemiological study of hemiplegia due to stroke in South India.," Stroke, vol. 1, no. 6, pp. 477-481, 1970.

[5] T. K. Banerjee, C. S. Mukherjee, and A. Sarkhel, "Stroke in the urban population of Calcutta: an epidemiological study," Neuroepidemiology, vol. 20, no. 3, pp. 201-207, 2001.

[6] D. Virok, Z. Kis, L. Karai et al., "Chlamydia pneumoniae in atherosclerotic middle cerebral artery," Stroke, vol. 32, no. 9, pp. 1973-1976, 2001.

[7] M. V. Kalayoglu, P. Libby, and G. I. Byrne, "Chlamydia pneumoniae as an emerging risk factor in cardiovascular disease," Journal of the American Medical Association, vol. 288, no. 21, pp. 2724-2731, 2002.
[8] G. K. Hansson, "Mechanisms of disease: inflammation, atherosclerosis, and coronary artery disease," The New England Journal of Medicine, vol. 352, no. 16, pp. 1626-1695, 2005.

[9] P. M. Ridker, C. H. Hennekens, J. E. Buring, R. Kundsin, and J. Shih, "Baseline IgG antibody titers to Chlamydia pneumoniae, Helicobacter pylori, herpes simplex virus, and cytomegalovirus and the risk for cardiovascular disease in women," Annals of Internal Medicine, vol. 131, no. 8, pp. 573-577, 1999.

[10] R. Cosentini and F. Blasi, "New pathogens for respiratory infections," Current Opinion in Pulmonary Medicine, vol. 2, no. 3, pp. 174-180, 1996.

[11] U. Waje-Andreassen, J. Kråkenes, E. Ulvestad et al., "IL-6: an early marker for outcome in acute ischemic stroke," Acta Neurologica Scandinavica, vol. 111, no. 6, pp. 360-365, 2005.

[12] F. Perini, M. Morra, M. Alecci, E. Galloni, M. Marchi, and V. Toso, "Temporal profile of serum anti-inflammatory and proinflammatory interleukins in acute ischemic stroke patients," Neurological Sciences, vol. 22, no. 4, pp. 289-296, 2001.

[13] M. A. Mendall, P. M. Goggin, N. Molineaux et al., "Relation of Helicobacter pylori infection and coronary heart disease," British Heart Journal, vol. 71, no. 5, pp. 437-439, 1994.

[14] P. J. Cook, D. Honeybourne, G. Y. H. Lip, D. G. Beevers, R. Wise, and P. Davies, "Chlamydia pneumoniae antibody titers are significantly associated with acute stroke and transient cerebral ischemia: the West Birmingham stroke project," Stroke, vol. 29, no. 2, pp. 404-410, 1998.

[15] M. Leinonen, "Pathogenetic mechanisms and epidemiology of Chlamydia pneumoniae," European Heart Journal, vol. 14, pp. 57-61, 1993.

[16] R. Jin, G. Yang, and G. Li, "Inflammatory mechanisms in ischemic stroke: role of inflammatory cells," Journal of Leukocyte Biology, vol. 87, no. 5, pp. 779-789, 2010.

[17] N. K. Rai, R. Choudhary, R. Bhatia et al., "Chlamydia pneumoniae seropositivity in adults with acute ischemic stroke: a casecontrol study," Annals of Indian Academy of Neurology, vol. 14, no. 2, pp. 93-97, 2011.

[18] Y. Sonobe, J. Liang, S. Jin et al., "Microglia express a functional receptor for interleukin-23," Biochemical and Biophysical Research Communications, vol. 370, no. 1, pp. 129-133, 2008.

[19] B. Becher, B. G. Durell, and R. J. Noelle, "IL-23 produced by CNS-resident cells controls $\mathrm{T}$ cell encephalitogenicity during the effector phase of experimental autoimmune encephalomyelitis," Journal of Clinical Investigation, vol. 112, no. 8, pp. 1186-1191, 2003.

[20] E. H. Lo, "Experimental models, neurovascular mechanisms and translational issues in stroke research," British Journal of Pharmacology, vol. 153, supplement 1, pp. S396-S405, 2008.

[21] K. Gertz, G. Kronenberg, R. E. Kälin et al., "Essential role of interleukin-6 in post-stroke angiogenesis," Brain, vol. 135, no. 6, pp. 1964-1980, 2012.

[22] Y. Maeda, M. Matsumoto, O. Hori et al., "Hypoxia/ reoxygenation-mediated induction of astrocyte interleukin 6: a paracrine mechanism potentially enhancing neuron survival," Journal of Experimental Medicine, vol. 180, no. 6, pp. 2297-2308, 1994.

[23] S. Suzuki, K. Tanaka, S. Nogawa et al., "Temporal profile and cellular localization of interleukin- 6 protein after focal cerebral ischemia in rats," Journal of Cerebral Blood Flow and Metabolism, vol. 19, no. 11, pp. 1256-1262, 1999.

[24] I. Gueorguieva, S. R. Clark, C. J. McMahon et al., "Pharmacokinetic modeling of interleukin- 1 receptor antagonist in plasma and cerebrospinal fluid of patients following subarachnoid 
hemorrhage," British Journal of Clinical Pharmacology, vol. 65, no. 3, pp. 317-325, 2008.

[25] D. Acalovschi, T. Wiest, M. Hartmann et al., "Multiple levels of regulation of the interleukin-6 system in stroke," Stroke, vol. 34, no. 8, pp. 1864-1869, 2003.

[26] G. Madre, J. L. Garcia, R. C. Gonzalez et al., "Association between seropositivity to Chlamydia pneumoniae and acute ischaemic stroke," European Journal of Neurology, vol. 9, no. 3, pp. 303-306, 2002.

[27] S. L. Melnick, E. Shahar, A. R. Folsom et al., "Past infection by Chlamydia pneumoniae strain and asymptomatic carotid atherosclerosis," The American Journal of Medicine, vol. 95, no. 5, pp. 499-504, 1993.

[28] S. L. Stevens, J. Bao, J. Hollis, N. S. Lessov, W. M. Clark, and M. P. Stenzel-Poore, "The use of flow cytometry to evaluate temporal changes in inflammatory cells following focal cerebral ischemia in mice," Brain Research, vol. 932, no. 1-2, pp. 110-119, 2002.

[29] D. L. Hahn, T. Anttila, and P. Saikku, "Association of Chlamydia pneumoniae IgA antibodies with recently symptomatic asthma," Epidemiology and Infection, vol. 117, no. 3, pp. 513-517, 1996.

[30] C. J. Smith, H. C. A. Emsley, C. M. Gavin et al., "Peak plasma interleukin-6 and other peripheral markers of inflammation in the first week of ischaemic stroke correlate with brain infarct volume, stroke severity and long-term outcome," $B M C$ Neurology, vol. 4, article 5, 2004.

[31] M. V. Kalayoglu, P. Libby, and G. I. Byrne, "Chlamydia pneumoniae as an emerging risk factor in cardiovascular disease," Journal of the American Medical Association, vol. 288, no. 21, pp. 2724-2731, 2002.

[32] P. Saikku, K. Mattila, M. S. Nieminen et al., "Serological evidence of an association of a novel chlamydia, with chronic coronary heart disease and acute myocardial infarction," The Lancet, vol. 2, no. 8618, pp. 983-986, 1988.

[33] S. Pelidou, N. Kostulas, D. Matusevicius, P. Kivisäkk, V. Kostulas, and H. Link, "High levels of IL-10 secreting cells are present in blood in cerebrovascular diseases," European Journal of Neurology, vol. 6, no. 4, pp. 437-442, 1999.

[34] V. C. S. S. Bandaru, V. Laxmi, M. Neeraja et al., "Chlamydia pneumoniae antibodies in various subtypes of ischemic stroke in Indian patients," Journal of the Neurological Sciences, vol. 272, no. 1-2, pp. 115-122, 2008.

[35] M. S. V. Elkind, I. F. Lin, J. T. Grayston, and R. L. Sacco, "Chlamydia pneumoniae and the risk of first ischemic stroke: the northern manhattan stroke study," Stroke, vol. 31, no. 7, pp. 1521-1525, 2000.

[36] E. Tarkowski, L. Rosengren, C. Blomstrand et al., "Early intrathecal production of interleukin- 6 predicts the size of brain lesion in stroke," Stroke, vol. 26, no. 8, pp. 1393-1398, 1995.

[37] J. S. Kim, S. S. Yoon, Y. H. Kim, and J. S. Ryu, "Serial measurement of interleukin-6, transforming growth factor- $\beta$, and S-100 protein in patients with acute stroke," Stroke, vol. 27, no. 9, pp. 1553-1557, 1996.

[38] W. Whiteley, C. Jackson, S. Lewis et al., "Inflammatory markers and poor outcome after stroke: a prospective cohort study and systematic review of interleukin-6," PLoS Medicine, vol. 6, no. 9, Article ID e1000145, 2009.

[39] J. Castillo and I. Rodríguez, "Biochemical changes and inflammatory response as markers for brain ischaemia: molecular markers of diagnostic utility and prognosis in human clinical practice," Cerebrovascular Diseases, vol. 17, supplement 1, pp. 7$18,2004$.
[40] N. Vila, J. Castillo, A. Dávalos, A. Esteve, A. M. Planas, and Á. Chamorro, "Levels of anti-inflammatory cytokines and neurological worsening in acute ischemic stroke," Stroke, vol. 34, no. 3, pp. 671-675, 2003.

[41] P. J. Martin, T. V. Enevoldson, and P. R. Humphrey, "Causes of ischemic stroke in young," Postgraduate Medical Journal, vol. 73, no. 855, pp. 8-16, 1997.

[42] G. Yilmaz and D. N. Granger, "Cell adhesion molecules and ischemic stroke," Neurological Research, vol. 30, no. 8, pp. 783793, 2008.

[43] N. Vila, J. Castillo, A. Dávalos, and Á. Chamorro, "Proinflammatory cytokines and early neurological worsening in ischemic stroke," Stroke, vol. 31, no. 10, pp. 2325-2329, 2000.

[44] J. Ngeh, S. Gupta, C. Goodbourn, B. Panayiotou, and G. McElligott, "Chlamydia pneumoniae in elderly patients with stroke (C-PEPS): a case-control study on the seroprevalence of Chlamydia pneumoniae in elderly patients with acute cerebrovascular disease," Cerebrovascular Diseases, vol. 15, no. 1-2, pp. 11-16, 2003.

[45] K. Yamashita, K. Ouchi, M. Shirai, T. Gondo, T. Nakazawa, and H. Ito, "Distribution of Chlamydia pneumoniae infection in the atherosclerotic carotid artery," Stroke, vol. 29, no. 4, pp. 773-778, 1998. 

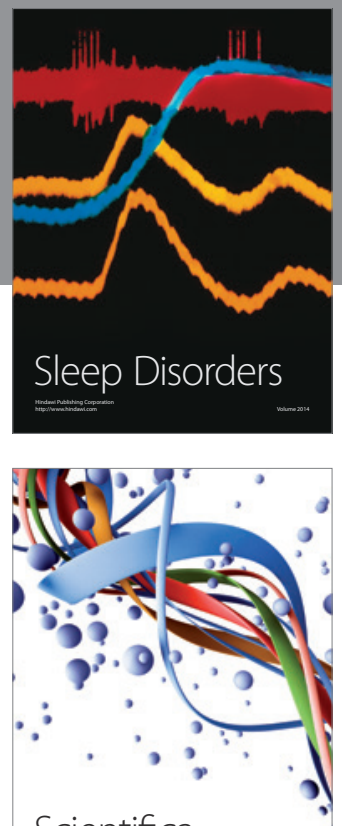

Scientifica
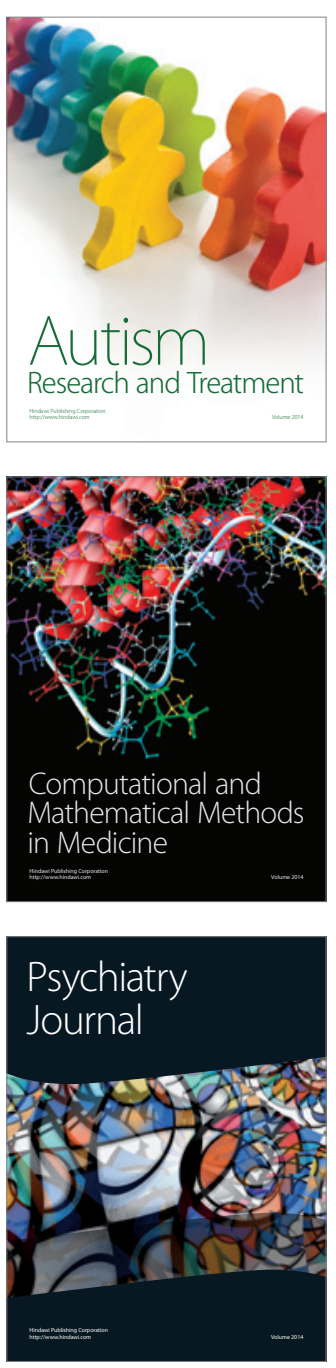
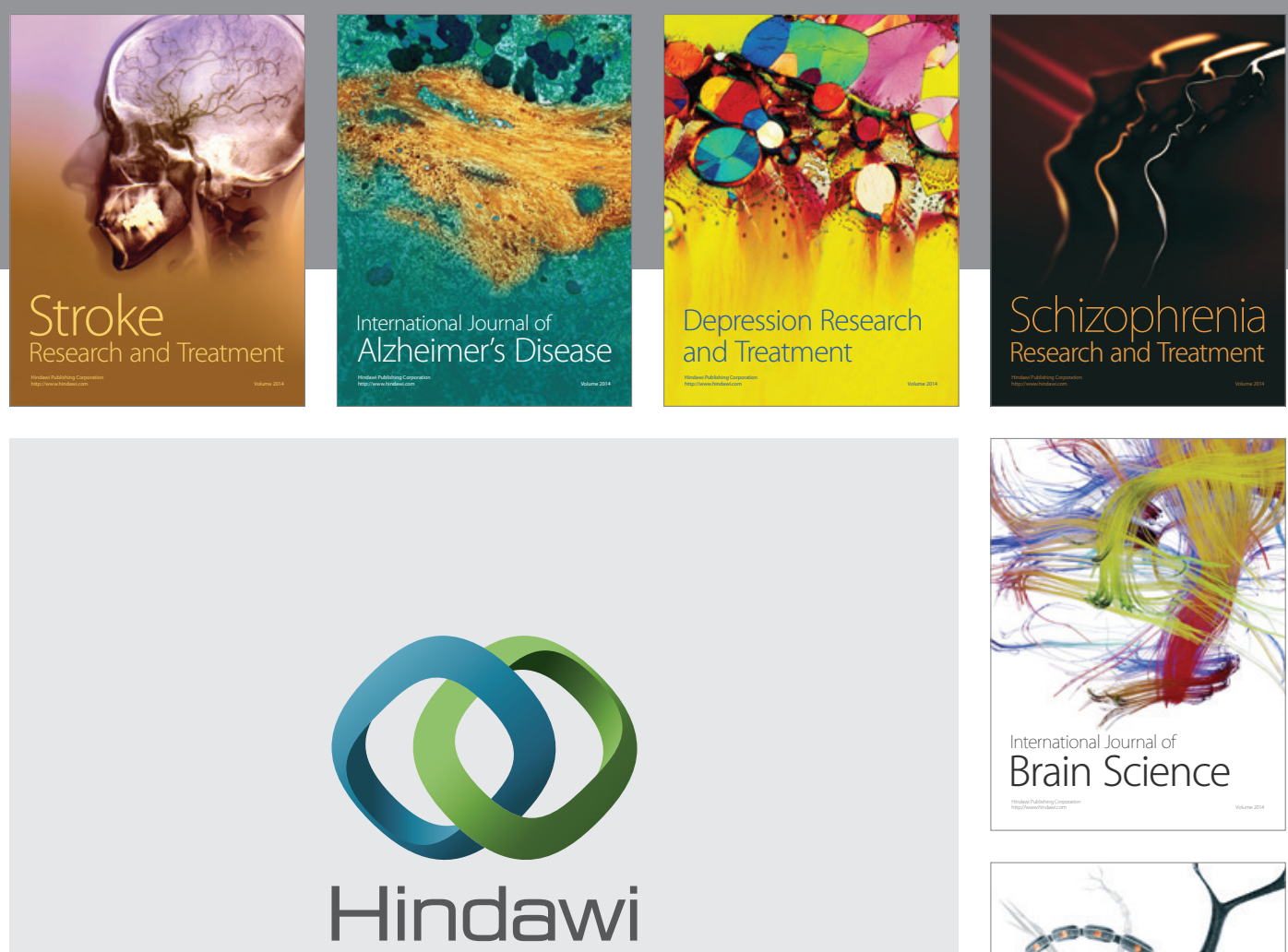

Submit your manuscripts at

http://www.hindawi.com
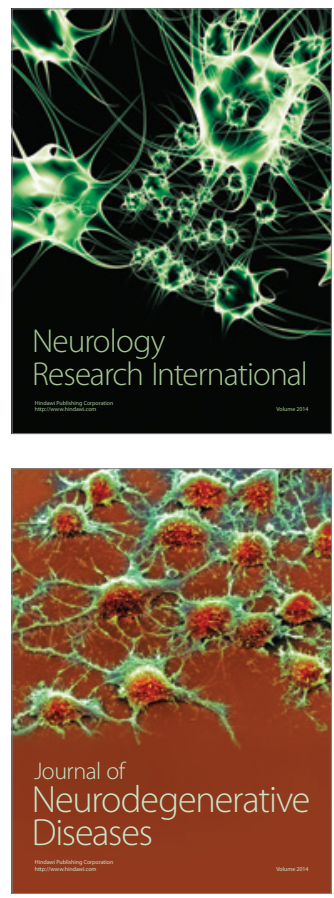

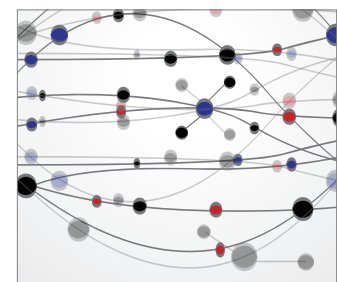

The Scientific World Journal
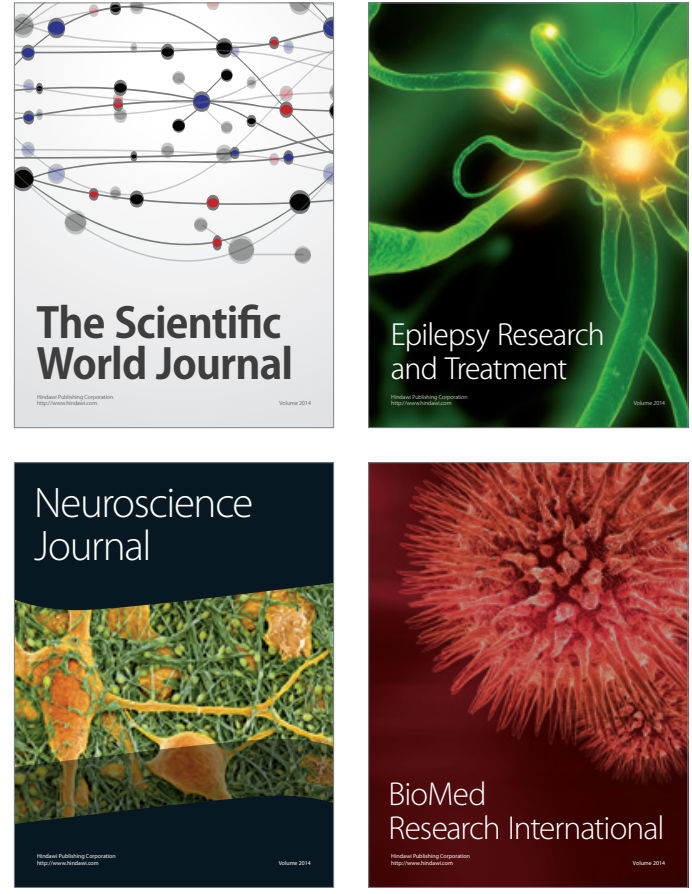

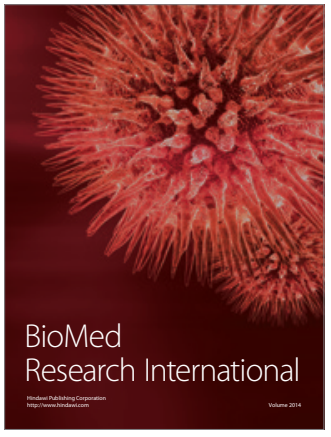

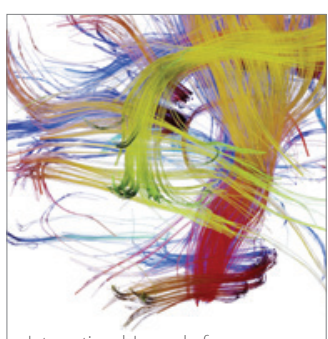

Brain Science

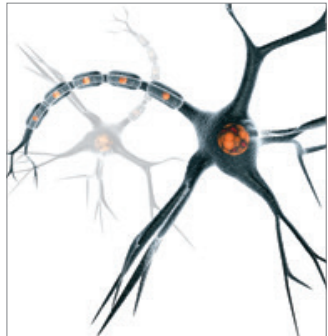

Neural Plasticity
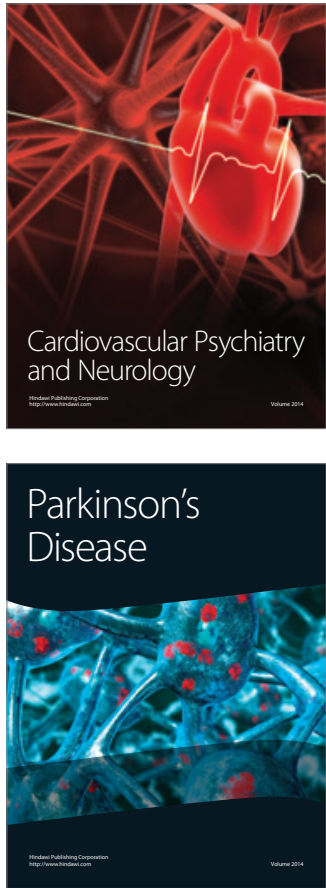